\title{
Variabilidad genética y detección de error en filiación utilizando microsatélites en dos rebaños de alpacas Huacaya (Vicugna pacos).
}

Genetic variability of Peruvian Alpacas (Vicugna pacos) by using microsatellite markers.

\author{
Claudia Yalta ${ }^{1}$, Giovanna Sotil ${ }^{2}$, Eudosio Veli ${ }^{1}$
}

\section{RESUMEN:}

Objetivo: Determinar la variabilidad genética y evaluar la utilidad de microsatélites (STR) en la determinación de paternidad en alpacas blancas huacaya, pertenecientes al Centro Piloto de Mejoramiento Genético Munay Paqocha y el Fundo Itita, de la Sociedad Peruana de Criadores de Alpacas y Llamas (SPAR) Puno. Realizar la genotipificación y selección de marcadores STR útiles para la asignación de paternidad y parentesco. Metodología: Se evaluaron 10 marcadores STR a partir de ADN aislado de sangre y de folículos pilosos de 183 individuos colectados al azar procedentes de dos rebaños. Resultados y Conclusiones: Se observó un alto nivel de variabilidad alélica en el total de individuos analizados, y la presencia de alelos exclusivos entre poblaciones, con frecuencias menores al 1,5\% en los loci LCA37, LCA90, LCA5, VOLP92, YWLL36, YWLL44 y YWLL08. Se propone la incorporación de tres marcadores adicionales, VOLP92, LCA94 y LCA90 para los análisis de variabilidad genética en alpacas. Los valores de $\mathrm{F}_{\mathrm{IS}}(0,016)$, y $\mathrm{F}_{\mathrm{ST}}(0,003)$ reflejaron bajo niveles de endogamia. El rebaño del Fundo Itita presentó una mayor Ho $(0,858)$ respecto a la He $(0,848)$, mientras que por el contrario el rebaño del Centro Munay Paqocha presentó un menor valor de la Ho $(0,815)$ respecto a la $\mathrm{He}(0,848)$, con una tendencia al déficit de heterocigotos. Los 10 marcadores presentaron una probabilidad de exclusión de parentesco adecuada, con un valor superior al 99,9\%, cuando se conoce el genotipo de ambos padres, y un poder de discriminación mayor a 0,90. Además, se alcanzó un valor PEC de 0,999 considerando solo los marcadores YWLL08, YWLL44, LCA37, YWLL36, LCA8; siendo YWLL08 el de mayor valor $(0,885)$. La prueba de filiación permitió detectar mayores errores de asignación de maternidad $(13,04 \%)$ y paternidad (30,4\%) en el rebaño del Fundo Itita, en comparación a Munay Paqocha con errores de designación de maternidad de 7,69\% y de paternidad de 17,95\%, concluyendo que este centro posee un mejor registro de empadres.

Palabras clave: camélidos sudamericanos, heterocigosidad, coeficiente de endogamia, probabilidad de exclusión, STR.

\section{ABSTRACT:}

Aim: To determine the genetic variability and the selection of STR markers useful for the evaluation of inbreeding, assignment of paternity and kinship, and the genotyping of two breeding herds of white huacayas alpacas Vicugna pacos, from the Pilot Center for Genetic Improvement Munay Paqocha and Fundo Itita, in Puno Perú. Methodology: 10 STR markers were assessed in 183 individuals, randomly selected. Results and Conclusions: We observed a high level of allelic variability in the total individuals, and unique alleles among

Laboratorio de Biología Molecular y Genómica. Dirección de Recursos Genéticos y Biotecnología, Instituto Nacional de Innovación Agraria INIA

2 Facultad de Ciencias Biológicas, Universidad Nacional Mayor de San Marcos. 
populations with frequencies lower than $1.5 \%$ in loci LCA37, LCA90, LCA5, VOLP92, YWLL36, YWLL44 and YWLL08. We propose the addition of three markers, VOLP92, LCA94 and LCA90 for the genetic variability analysis in alpacas. $\mathrm{F}_{\mathrm{IS}}(0.016)$ and $\mathrm{F}_{\mathrm{ST}}(0.003)$ values reflected low levels of inbreeding. Fundo Itita herd showed higher Ho (0.858) than He (0.848), while the herd of Munay Paqocha showed lower Ho (0.815) respect to $\mathrm{He}(0.848)$, with trending heterozygote deficit. The 10 markers showed an appropriate exclusion relationship probability, with a value greater than $99.9 \%$ when the genotype of both parents was known, and a power of discrimination greater than 0.9. In addition, a PEC value of 0.999 was reached considering only markers YWLL08, YWLL44, LCA37, YWLL36, LCA8; YWLL08 being the highest value (0.885). Filiation test detected major errors of maternity (13.04\%) and paternity (30.4\%) in the herd Fundo Itita. In Munay Paqocha with respect to errors designation maternity $7.69 \%$ and paternity of $17.95 \%$, concluding that Munay Paqocha had a better record of matings.

Keywords: STR, camelids, heterozygosity, inbreeding coefficient, probability of exclusion.

\section{INTRODUCCIÓN}

La alpaca Vicugna pacos es uno de los cuatro camélidos sudamericanos más importante desde el punto de vista económico y social, ya que sirven de sustento para las familias de las comunidades alto andinas (FAO, 2005). El recurso principalmente aprovechado es la fibra, sin embargo las deficiencias en los sistemas de crianza tradicional han contribuido con la disminución de la calidad productiva de los animales (peso, talla, peso de vellón y finura de fibra), volviéndolos menos competitivos en el mercado internacional. Debido a esto, las asociaciones de criadores han incrementado las iniciativas para el desarrollo de programas de mejora genética, orientadas al blanqueamiento y finura de fibra. No obstante, a la fecha se tienen pocos avances, debido principalmente a la sobreestimación de los valores de cría producto de las deficiencias en la identificación de los animales y la falta de adecuados registros genealógicos. En este sentido, se considera necesario que los animales seleccionados para el empadre deban de someterse a la verificación de paternidad de su progenie a fin de obtener predicciones confiables en la estimación de caracteres cuantitativos, por lo que se requiere de la organización de bases de datos (a nivel genético molecular), con una estructura de información completa y confiable del pedigrí para evitar apareamientos entre individuos emparentados (endogamia). Por otro lado, los inadecuados registros genealógicos conducen a un incremento considerable en los estimadores de correlación genética (FAO, 2010; Velarde, 2011); así, la adquisición de un progenitor cuyos valores genéticos aditivos como peso de nacimiento, cantidad de vellón, entre otros, se encuentran sobreestimados y puede conducir a la disminución de la ganancia genética del rebaño.

En este contexto, el conocer la variabilidad genética poblacional es una condición necesaria para el mejoramiento de los caracteres de interés. Se ha encontrado que la disminución de la variabilidad genética dada por el aumento del nivel de endogamia puede causar la expresión de defectos congénitos y hereditarios, muy frecuentes en rebaños de fibra blanca, tales como prognatismo, polidactilia, ojos zarcos (Briones y Valdivia, 1985; Huanca et al., 2007).

Los marcadores moleculares basados en el análisis del ADN han permitido solucionar estos problemas de asignación y verificación de progenitores, así como en la evaluación de la variabilidad genética y el monitoreo de los niveles de endogamia. Los microsatélites (STRs) son secuencias cortas repetitivas, distribuidas en todo el genoma que, debido a su alta tasa de mutación y su naturaleza codominante, permiten la estimación de la diversidad genética entre y dentro de poblaciones. Diversos autores reportan el uso de STRs en la estimación de la diversidad genética, identificación de individuos, control de filiación en diferentes especies domésticas de importancia económica, como en ovejas (Pariset et al., 2003, Tolone et al., 2012), bovinos (Chikhi et al., 2004; Egito et al., 2007), cerdos (Laval et al., 2000); para garantizar la fiabilidad de los documentos genealógicos (Jianlin et al., 2000), con fines de conservación y mejora genética (Mburu, 2003). Además de los estudios de diversidad genética, los análisis de paternidad 
utilizando microsatélites son extensamente usados como una herramienta de apoyo en el mejoramiento genético. Se ha descrito que con un total de 10 microsatélites en alpacas, la probabilidad de exclusión supera al 0,999 (Agapito et al., 2008), e incluso la posibilidad de utilizarlos en casos de tráfico ilegal entre especies domésticas (Marín et al., 2009; Di Rocco et al., 2011).

Bajo este contexto, en el distrito de CarabayaProvincia de Macusani, la Sociedad Peruana de Criadores de Alpacas y Llamas (SPAR) de Macusani, y con el apoyo de la Oxfam en el 2006, iniciaron un proyecto de mejoramiento genético de la finura de fibra, creando el Centro Munay Paqocha y el Fundo Itita. En este sentido, la selección de alpacas huacayas blancas obtenidas de las diferentes comunidades de la Región de Puno, se realizó considerando sus características fenotípicas (finura de fibra) para determinar qué individuos participarían en el empadre con el fin de obtener un núcleo reproductor élite. Es conocido que estos procesos de selección requieren del conocimiento de la variabilidad genética poblacional y de la determinación de paternidad en la medida en que pueden servir de apoyo para los cálculos de índices de selección más precisos.

En tal sentido, el presente trabajo buscó determinar la variabilidad genética y realizar la genotipificación de dos rebaños de reproductores de alpacas huacayas blancas pertenecientes al Centro Piloto de Mejoramiento Genético Munay Paqocha y el Fundo Itita, de la Sociedad Peruana de Criadores de Alpacas y Llamas (SPAR) Macusani. Además se buscó identificar el nivel de endogamia, y realizar la selección de marcadores STR que sirvan de herramienta para la determinación de pruebas de paternidad y parentesco a fin de contribuir en programas de mejoramiento genético de camélidos sudamericanos.

\section{MATERIALES Y MÉTODOS}

Se recolectaron muestras de sangre y de folículos pilosos, de un total de 183 alpacas de raza Huacaya blancas, procedentes del Centro Piloto de Mejoramiento Genético Munay Paqocha (14 ${ }^{\circ}$ ' 40 " LS y $70^{\circ} 21^{\prime}$ 00" LO) ubicado en el distrito de Macusani a $4633 \mathrm{msnm}$, y del Fundo Itita $\left(14^{\circ} 11^{\text {' }}\right.$ 18 " LS y $70^{\circ} 17^{\prime} 24$ " LO) ubicado en el distrito de
Ajoyani a $4585 \mathrm{msnm}$.

Las extracciones de ADN genómico se realizaron según el método de Sambrook y Russell (1989), y Burgos-Paz et al. (2010). Para la amplificación de los microsatélites, se seleccionaron 10 marcadores genéticos fluoromarcados (Tabla 1) descritos en estudios previos de camélidos (Lang et al., 1996; Penedo et al., 1998; Obreque et al., 1998; Penedo et al., 1999; Obreque et al., 1999; Jianlin et al., 2000). Las reacciones de amplificación por PCR multiplex y simples se prepararon en un volumen final de 10 $\mu \mathrm{l}$, considerando las siguientes concentraciones finales: $1 \mathrm{X}$ buffer de PCR, $\mathrm{MgCl}_{2} 2,0 \mathrm{mM}$, dNTP $0,25 \mathrm{mM}$, cebador $0.1-0,2 \mu \mathrm{M}, 0,05 \mathrm{U}$ de Taq Polimerasa, y $2 \mu 1$ de ADN $25 \mathrm{ng} / \mu \mathrm{l}$. La PCR se realizó en un termociclador Mastercycler ${ }^{\circledR}$ Pro Eppendorf, utilizando el siguiente programa de temperaturas: una fase de desnaturalización a $95{ }^{\circ} \mathrm{C}$ por 5 minutos, seguida de 25 ciclos con una primera fase de desnaturalización a $95^{\circ} \mathrm{C}$ por 30 segundos, hibridación o anillamiento del iniciador a $61{ }^{\circ} \boldsymbol{C} y 57$ ${ }^{\circ} \mathrm{C}$ por 90 segundos, y una de fase extensión a $72{ }^{\circ} \mathrm{C}$ por 1 minutos; además de una extensión final a 60 ${ }^{\circ} \mathrm{C}$ por 30 minutos.

Los productos de PCR fueron separados en un analizador genético ABI 3130XL (Applied Biosystems, Foster City, CA, USA) mediante electroforesis capilar, utilizando el gel polímero POP 7 (Performance Optimised Polymer). Cada 1,5 $\mu 1$ de producto de PCR fueron mezclados con 9,75 ul de formamida Hi-Di y $0.25 \mu l$ de un marcador estándar interno de $500 b p$ GeneScan ROX ${ }^{\mathrm{TM}}$. Luego las muestras fueron sometidas en $94{ }^{\circ} \mathrm{C}$ durante 3 min para denaturación e inmediatamente se produjo un choque térmico colocándolo 3 min en hielo antes de realizar la corrida en el analizador genético.

Para el análisis de los fragmentos se utilizó el programa GENEMAPPER v. 4.0 Applied Biosystems, a fin de asignar los alelos a los fragmentos identificados. Para el análisis de la variabilidad genética se estimó el número efectivo de alelos (Ae) según Frankham et al. (2002), el número de alelos y las frecuencias alélicas. La distribución de las frecuencias alélicas de cada locus en cada rebaño reproductor fueron graficadas en histogramas. La evaluación del equilibrio de Hardy-Weinberg (EHW) para los 10 marcadores microsatélites se realizó según la prueba exacta de Fisher, utilizando el método de cadena de Monte 
Carlo Markov (Guo y Thompson,1992), así como el déficit y exceso de heterocigotos, además del desequilibrio de ligamiento con el programa GENEPOP v. 4.1 (Rousset, 2008). La corrección de Bonferroni se aplicó para obtener el valor crítico para múltiples pruebas sobre los 10 loci por población. Los índices de Fijación $\left(\mathrm{F}_{\mathrm{IS}}, \mathrm{F}_{\mathrm{ST}}\right.$, $\mathrm{F}_{\text {IT }}$ ) fueron calculados usando el programa FSTAT versión 2.9.3.2 (Goudet, 1995). El contenido de índice Polimórfico (PIC), las heterocigosidades observada (Ho) y esperada $(\mathrm{He})$ para cada uno de los loci y las heterocigosidades totales fueron determinadas con el programa CERVUS v. 3.0 (Kalinowski, et al., 2007).

El poder de discriminación (PD), fue calculado con el programa Power Stat v. 12; la probabilidad de exclusión (PE) de los marcadores STR se determinó con el programa CERVUS v. 3.0 (Kalinowski et al., 2007) bajo tres supuestos: primero es la probabilidad de exclusión cuando uno de los genotipos (paternal o maternal) no está disponible (PE-1), segundo cuando se conoce los genotipos del padre, madre y cría (PE-2), y por último cuando se tiene un par de padres candidatos (PE-PP) (Garber y Morris et al., 1983).

Se realizó un análisis de identidad, para encontrar coincidencias o duplicidad de genotipos. La simulación de paternidad tiene dos propósitos, evaluar el poder de resolución de los marcadores y estimar el valor crítico del LOD (logaritmo natural de verosimilitud -odd ratio) para determinar el nivel de confidencia. Además, se realizó una simulación para determinar el grado de confianza de los marcadores microsatélites, considerando 10000 ciclos para 75 padres candidatos y un error de tipificación de 0,01 .

Finalmente, para la designación de paternidad se realizaron simulaciones de paternidad/maternidad con dos intervalos de confianza de $80 \%$ y $95 \%$, las cuales corresponden a condiciones relajadas y estrictas, respectivamente. Se calcularon los LOD y los valores delta $(\Delta)$ para cada uno de los machos adultos, según Marshall et al. (1998).Los resultados se evaluaron de acuerdo a los valores de delta $(\Delta)$ y el LOD. El valor delta es la diferencia de los valores de LOD de los padres más probables; un valor positivo implica que el padre designado es el más probable, $\mathrm{y}$ un valor negativo ocurre cuando el padre en cuestión no es compatible. Para el análisis se formaron dos grupos de crías: 39 familias de Munay Paqocha y 23 familias del Fundo Itita, utilizando como base los registros genealógicos proporcionados por el SPAR-Macusani.

\section{RESULTADOS}

\section{Estimación de parámetros de variabilidad genética.}

El número total de alelos (A) identificados a través de los 10 marcadores STR fue de 134. El

Tabla 1. Número total de alelos por rebaño de alpacas del Centro Munay Paqocha (MP) y del Fundo Itita

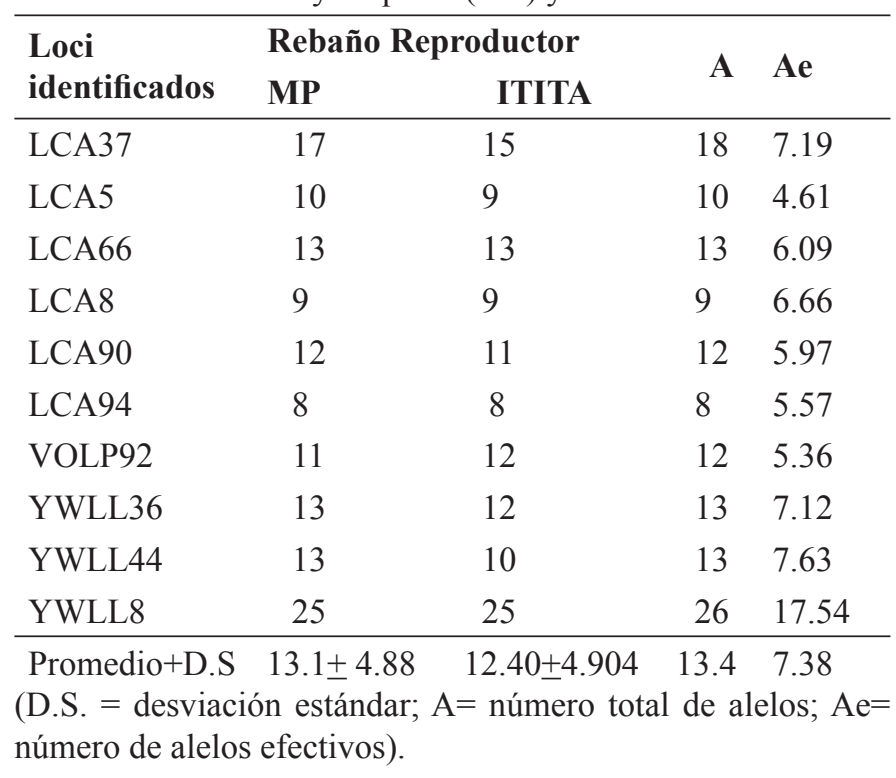


mayor número de alelos se encontró en el locus YWLL $08(n=26)$ representando el 19,4\% del total; mientras el menor número $(\mathrm{n}=8)$ para el locus LCA8 (5,97\% del total). Los individuos del Centro Munay Paqocha presentaron un promedio de 13,1 alelos, mientras que de Itita un promedio de 12,4 alelos, ambas zonas con similares valores de desviaciones estándar (Tabla 1).

Los alelos 166 (LCA37), 209 (VOLP92) y 141 (YWLL44) fueron exclusivos para el fundo Itita, y los alelos 128,148, 154 (LCA37), 200 (LCA05), 263

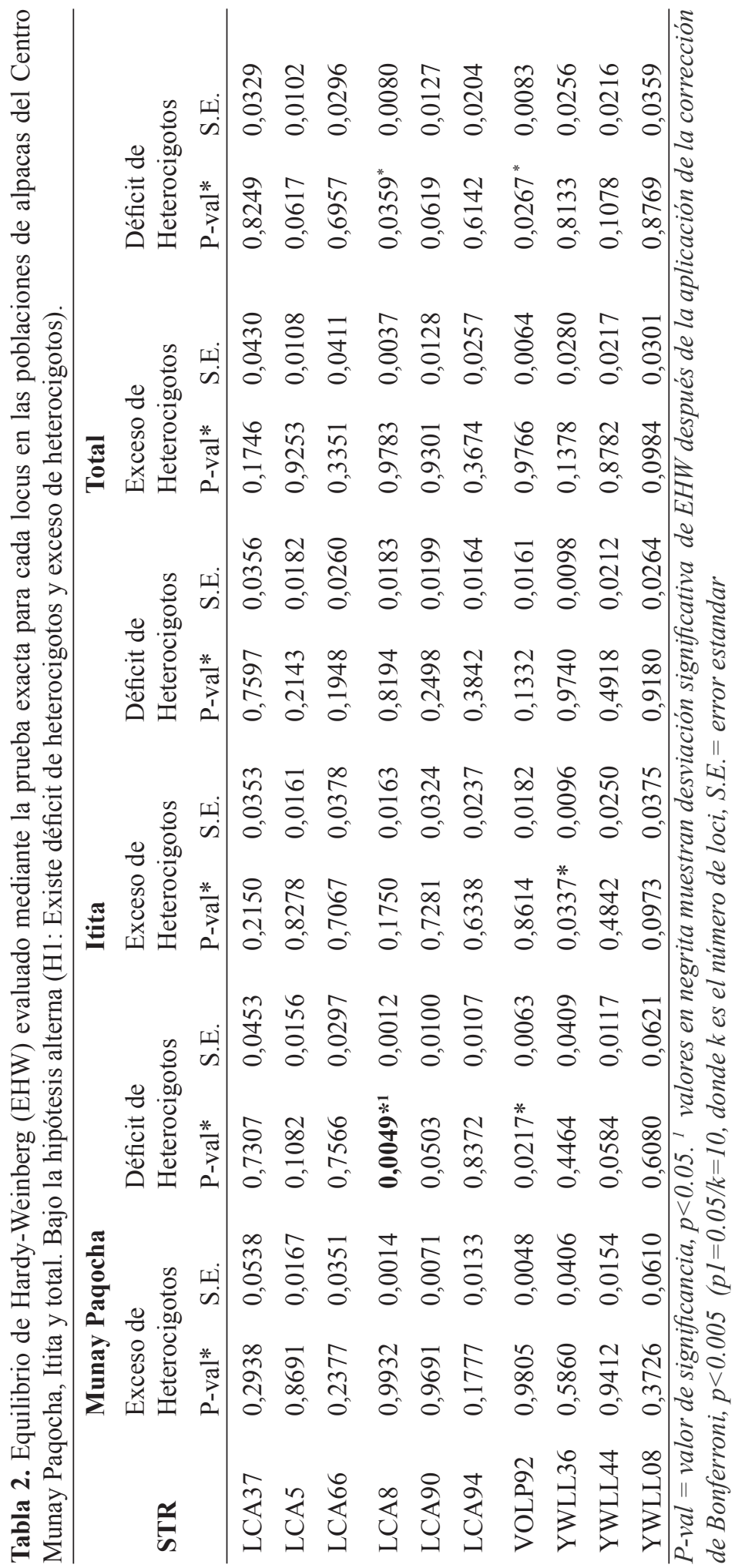


(LCA90), 157 (YWLL36), 90, 114,124 (YWLL44), 131 (YWLL08) para el centro Munay Paqocha con frecuencias mayores a 0,05 y menores a 0.15 .

Después de aplicar la corrección de Bonferroni, tomando en cuenta todos los marcadores utilizados, fueron no significativos para el desequilibrio de ligamiento $(\mathrm{p}<0.00111)$. La prueba global para los dos rebaños y los 10 loci analizados mostró que los loci LCA8 y VOLP 92 se encontraron en EHW después de aplicar la corrección de Bonferroni ( $p<0,005$; Rice, 1989), obteniéndose valores de $p$ $=0.0359$ y $\mathrm{p}=0,0267$, respectivamente. Cuando se realizó el análisis por rebaño reproductor, en el Centro Munay Paqocha se observó una desviación del EHW para los loci LCA8 y VOLP92, y en el Fundo Itita se obtuvo desviación del loci YWLL36 (bajo la hipótesis alterna exceso de heterocigotos). Después de aplicar la corrección de Bonferroni en ambos análisis todos los loci mostraron equilibrio de Hardy y Weinberg (Tabla 2).

Los valores de heterocigosidad esperada $(\mathrm{He}) \mathrm{de}$ los marcadores LCA5, LCA8, LCA90, VOLP92, YWLL36, YWLL44 en el Centro Munay Paqocha fueron más altos que la observada (Ho), indicando un déficit de heterocigosidad; el Fundo Itita presentó 4 marcadores (LCA5, LCA90, LCA94, YWLL44) con un He mayor a la Ho, mientras que para ambos rebaños el LCA5 presentó el valor más bajo (0.777 y 0.796 respectivamente) y YWLL08 el valor más alto $(0,950$ y 0,937 , respectivamente)
Al analizar a los dos rebaños como uno solo, 6 marcadores presentaron valores de He mayores a los observados, indicando nuevamente un déficit de heterocigotos. Todos los marcadores STR mostraron un alto grado de heterocigosidad observada (Ho) con valores superiores a 0,732. Del mismo modo, la heterocigosidad esperada $(\mathrm{He})$ para cada loci presentaron valores superiores a 0,785 indicando una elevada diversidad genética. El valor de PIC, que determina el grado de polimorfismo de un marcador, para este grupo de loci se observó valores mayores a 0.5 entre 0,752 (LCA05) y 0,94 (YWLL08), con un promedio total de 0,8301 (Tabla 3)

Al analizar por población, el valor absoluto del $F_{\text {IS }}$ para ambas poblaciones no fue significativamente diferente de cero. El Fundo Itita presentó un $F_{I S}$ total negativo $(-0,012)$ mostrando exceso de heterocigotos (LCA37, LCA66, LCA8, VOLP92, YWLL36, YWLL08), mientras que por el contrario el Centro Munay Paqocha presentó solo dos marcadores con valores negativos (LCA37, LCA94), con un $F_{I S}$ para el total de 0,039. Para el total de la población, el valor $F_{S T}$ resultó ser muy bajo $(0,003)$, indicando una baja diferenciación genética entre las dos poblaciones; el $F_{I S}$ total mostró que la reducción en la heterocigosidad individual fue de 0.016, lo que indica un apareamiento aleatorio; el valor promedio de $F_{I T}$ que mide la disminución de la heterocigosidad de un individuo en relación a la población total fue de 0,019 (Tabla 4).

Tabla 3. Estimación de heterocigosidad de Nei 1984, PIC, calculadas para las poblaciones de alpacas del Fundo Itita, Centro Munay Paqocha y total.

\begin{tabular}{|c|c|c|c|c|c|c|c|}
\hline \multirow{2}{*}{ STR } & \multicolumn{2}{|c|}{ MUNAY PAQOCHA } & \multicolumn{2}{|c|}{ ITITA } & \multicolumn{2}{|c|}{ TOTAL } & \multirow{2}{*}{ PIC } \\
\hline & Ho & $\mathrm{He}$ & Ho & $\mathrm{He}$ & Ho & $\mathrm{He}$ & \\
\hline LCA37 & 0,870 & 0,865 & 0,892 & 0,864 & 0,880 & 0,863 & 0,848 \\
\hline LCA5 & 0,700 & 0,777 & 0,771 & 0,796 & 0,732 & 0,785 & 0,752 \\
\hline LCA66 & 0,800 & 0,804 & 0,867 & 0,860 & 0,831 & 0,838 & 0,820 \\
\hline LCA8 & 0,810 & 0,868 & 0,831 & 0,830 & 0,820 & 0,852 & 0,833 \\
\hline LCA90 & 0,760 & 0,842 & 0,807 & 0,827 & 0,781 & 0,834 & 0,813 \\
\hline LCA94 & 0,850 & 0,817 & 0,819 & 0,831 & 0,836 & 0,823 & 0,796 \\
\hline VOLP92 & 0,750 & 0,815 & 0,843 & 0,812 & 0,792 & 0,816 & 0,799 \\
\hline YWLL36 & 0,840 & 0,867 & 0,916 & 0,858 & 0,874 & 0,862 & 0,845 \\
\hline YWLL44 & 0,820 & 0,875 & 0,855 & 0,864 & 0,836 & 0,871 & 0,855 \\
\hline YWLL08 & 0,950 & 0,950 & 0,976 & 0,936 & 0,962 & 0,946 & 0,940 \\
\hline Promedio & $0,815 \pm 0,070$ & $0,848 \pm 0,049$ & $0,858 \pm 0,059$ & $0,848 \pm 0,039$ & $0,8344 \pm 0,063$ & $0,849 \pm 0,043$ & 0,8301 \\
\hline
\end{tabular}

$\mathrm{Ho}=$ heterocigosidad observada, $\mathrm{He}=$ heterocigosidad esperada, $\mathrm{PIC}=$ Contenido de información polimórfica 3

(D.S. = desviación estándar; $\mathrm{A}=$ número total de alelos; $\mathrm{Ae}=$ número de alelos efectivos). 


\section{Estimación de parámetros forenses y de paternidad}

Los estadísticos forenses miden en conjunto la confiabilidad al momento de diferenciar genéticamente a los individuos de una población. Al analizar la probabilidad de discriminación (PD) que permite evaluar la capacidad de los marcadores de discriminar a un individuo tomado al azar, se encontró que el locus con mayor poder de discriminación fue el YWLL08 (98.9\%). Igualmente los marcadores restantes presentaron valores superiores al 90\%. Los resultados de las probabilidades de exclusión mostraron altos valores (mayores al 0,999) en las tres situaciones. Además,

Tabla 4. Índice de Fijación $\left(\mathrm{F}_{\mathrm{IS}}, \mathrm{F}_{\mathrm{IT}}, \mathrm{F}_{\mathrm{ST}}\right)$ calculadas para la población de Total, Fundo Itita y el Centro Munay Paqocha evaluado mediante marcadores STR.

\begin{tabular}{lllll}
\hline $\begin{array}{l}\text { STR } \\
\text { MUNAY PAQOCHA }\end{array}$ & \multicolumn{2}{l}{$\begin{array}{l}\text { Rebaño } \\
\text { ITITA }\end{array}$} & TOTAL \\
\hline $\begin{array}{l}\text { Fis por locus por } \\
\text { población }\end{array}$ & LCA37 & $-0,006$ & $-0,033$ & $-0,019$ \\
& LCA5 & 0,099 & 0,032 & 0,067 \\
& LCA66 & 0,006 & $-0,009$ & 0,009 \\
& LCA8 & 0,067 & $-0,002$ & 0,038 \\
& LCA90 & 0,098 & 0,024 & 0,063 \\
& LCA94 & $-0,04$ & 0,014 & $-0,016$ \\
& VOLP92 & 0,08 & $-0,039$ & 0,029 \\
& YWLL36 & 0,031 & $-0,068$ & $-0,014$ \\
& YWLL44 & 0,063 & 0,01 & 0,04 \\
& YWLL08 & 0 & $-0,043$ & $-0,017$ \\
Total por población & $F_{I S}$ & 0,039 & $-0,012$ & 0,016 \\
& $F_{I T}$ & & & 0,019 \\
& $F_{S T}$ & & & 0,003 \\
\hline
\end{tabular}

Tabla 5. Probabilidades de exclusión combinada para los 10 microsatélites.

\begin{tabular}{lllll}
\hline STR & PE1 & PE-2 & PE-PP & PD \\
\hline LCA37 & 0,577 & 0,733 & 0,897 & 0,961 \\
LCA5 & 0,407 & 0,584 & 0,77 & 0,922 \\
LCA66 & 0,522 & 0,689 & 0,868 & 0,952 \\
LCA8 & 0,536 & 0,701 & 0,869 & 0,955 \\
LCA90 & 0,505 & 0,674 & 0,852 & 0,947 \\
LCA94 & 0,472 & 0,646 & 0,822 & 0,937 \\
VOLP92 & 0,49 & 0,664 & 0,856 & 0,946 \\
YWLL36 & 0,562 & 0,721 & 0,885 & 0,960 \\
YWLL44 & 0,579 & 0,736 & 0,895 & 0,964 \\
YWLL08 & 0,793 & 0,885 & 0,977 & 0,989 \\
PEC & 0,99972 & 0,9999966 & 0,999999999957 & \\
\hline
\end{tabular}

(PE-1 = cuando se conoce el genotipo de uno de los padres; PE-2 = cuando se conoce los genotipos de ambos padres la probabilidad de exclusión; PE-PP = cuando se tiene un par de padres candidatos la probabilidad de exclusión) 
Tabla 6. Probabilidad de exclusión combinada (PEC) en términos de número de microsatélites usados ordenados de mayor a menor PE

\begin{tabular}{|c|c|c|c|c|c|c|c|c|c|c|}
\hline STR & $1 *$ & $2^{*}$ & $3 *$ & $4 *$ & $5^{*}$ & $6^{*}$ & $7 *$ & $8^{*}$ & 9* & $10 *$ \\
\hline YWLL08 & 0,885 & 0,885 & 0,885 & 0,885 & 0,885 & 0,885 & 0,885 & 0,885 & 0,885 & 0,885 \\
\hline YWLL44 & & 0,736 & 0,736 & 0,736 & 0,736 & 0,736 & 0,736 & 0,736 & 0,736 & 0,736 \\
\hline LCA37 & & & 0,729 & 0,729 & 0,729 & 0,729 & 0,729 & 0,729 & 0,729 & 0,729 \\
\hline YWLL36 & & & & 0,721 & 0,721 & 0,721 & 0,721 & 0,721 & 0,721 & 0,721 \\
\hline LCA8 & & & & & 0,701 & 0,701 & 0,701 & 0,701 & 0,701 & 0,701 \\
\hline LCA66 & & & & & & 0,689 & 0,689 & 0,689 & 0,689 & 0,689 \\
\hline LCA90 & & & & & & & 0,674 & 0,674 & 0,674 & 0,674 \\
\hline VOLP92 & & & & & & & & 0,664 & 0,664 & 0,664 \\
\hline LCA94 & & & & & & & & & 0,642 & 0,642 \\
\hline LCA5 & & & & & & & & & & 0,584 \\
\hline PEC & 0,885 & 0,96964 & 0,991772 & 0,997705 & 0,999314 & 0,999787 & 0,99993 & 0,999977 & 0,999992 & 0,999997 \\
\hline
\end{tabular}

se observó la probabilidad de exclusión con menor valor para el LCA5 y mayor para el YWLL08, en las tres situaciones. El número de loci mínimo a utilizar para alcanzar una probabilidad mayor a 0,99 fue 5 con los PE-2 más altos (Tabla 6).

\section{Asignación y simulación de paternidad / maternidad}

Los resultados del análisis de identidad mostraron que un individuo fue colectado por duplicado. Se logró el asignamiento de paternidad del 100\% de las crías. De las 39 crías en el Centro Munay Paqocha, se encontró un 7,69\% de error de maternidad (LOD= $-2,93 \mathrm{E}+00--8,45 \mathrm{E}+00)$ y el error de paternidad fue $17,95 \%(\mathrm{LOD}=-1,33 \mathrm{E}+01$ a $-3.48 \mathrm{E}+00)$. Por otro lado, de las 23 crías en el Fundo Itita se encontraron $13.04 \%$ asignaciones maternas incorrectas $(\mathrm{LOD}=$ $-1,31 \mathrm{E}+01$ a $-3,77 \mathrm{E}+01)$ y un $30,4 \%$ de asignación paterna erróneas $(\mathrm{LOD}=-1,31 \mathrm{E}+01 \mathrm{a}-2,69 \mathrm{E}+01)$.

\section{DISCUSIÓN}

En los programas de mejoramiento genético animal, el efectuar cruces dirigidos permite formar rebaños homogéneos con características productivas similares. Tal es el caso del Centro Piloto de Mejoramiento Genético Munay Paqocha y el Fundo
Itita, cuya formación tuvo como finalidad generar núcleos de reproductores de alpacas huacayas blancas orientadas a obtener mejoras en la finura de la fibra generando la reducción del tamaño efectivo de la población produciendo la disminución del número de alelos, especialmente la pérdida de aquellos que se encuentran en frecuencias muy bajas debido al efecto de la deriva génica, en este trabajo no se detectaron perdidas de alelos.

Los 10 marcadores utilizados en este trabajo mostraron un alto nivel de variabilidad alélica, de los cuales 7 fueron descritas previamente por Agapito et al. (2008) como útiles para los estudios de variabilidad, mientras que los otros tres marcadores VOLP92, LCA94, LCA90 que fueron descritos previamente para estudios en camellos (Schulz et al., 2005) y propuesto en este estudio, también mostraron ser útiles debido a su alto nivel de heterocigosidad observado en la población.

Si bien entre los rebaños no se observaron diferencias en el número de alelos, sin embargo estos valores sí fueron mayores comparado a lo descrito por La Manna et al. (2011) y Paredes et al. (2013), aunque menor a los descritos por Agapito et al. (2008), para los loci YWLL36, LCA5, LCA66, LCA08, YWLL44, LCA37, YWLL08 atribuido probablemente al mayor número de individuos 
analizados por población $(\mathrm{n}=329)$. De igual forma Barreta et al. (2012) informó que los marcadores con un mayor número de alelos fueron YWLL36, LCA5, LCA66, YWLL44 analizados en poblaciones de alpacas bolivianas $(n=149)$. La diferencias entre los números de alelos encontrados en otros estudios y los analizados en el presente trabajo expresa una tendencia particular de algunos de los microsatélites empleados a mutar más fácilmente $\mathrm{y}$, de este modo, generar un número más alto de alelos tal como fue descrito para el locus YWLL08, con 26 alelos. El conocimiento de las frecuencias alélicas poblacionales permite compararlas, así como el detectar la pérdida de la variabilidad y de posibles introgresiones o hibridizaciones entre especies (Kadwell et al., 2001).

Ambos rebaños presentaron alelos exclusivos aunque con frecuencias muy bajas $(<0.15)$, debido probablemente a su reciente origen, ocasionado ya sea por la pérdida (deriva genética) o ganancia de pares de bases (mutación).Así por ejemplo, para el marcador LCA37 la población de Munay Paqocha presentó los alelos exclusivos 128, 148, 154, mientras que el Fundo Itita presentó el alelo 166. La Manna et al. (2011) también reportó para este locus la presencia de un alto número de alelos exclusivos $(130,138,148,170)$ para la raza suri. Por otro lado, Bustamante et al. (2002) informaron sobre la presencia de alelos exclusivos para los loci YWLL08, YWLL36 en poblaciones argentinas de guanacos y llamas, mientras que en este estudio se registraron para el locus YWLL08 (131 para el Fundo Itita y 141 para el Centro Munay Paqocha). No obstante, es recomendable seguir identificando estos alelos en otras poblaciones y descartar si el tamaño de muestra resulta un parámetro importante en esta exclusividad a fin de considerarlos al momento de diseñar programas de mejoramiento y/o de conservación del material genético.

Existe una relación positiva entre el número de alelos efectivos, el nivel de polimorfismo (PIC) y heterocigosidad, registrado en este estudio. La Manna et al. (2011) también encontraron esta relación para 7 marcadores, de los 13 analizados, en poblaciones de alpacas procedentes de un centro de conservación. Además, todos los loci se encontraron en EHW Munay Paqocha, luego de aplicar la corrección de Bonferroni, que es utilizada para corregir falsos positivos en pruebas múltiples. además, contrastándolo con el valor de Fis (0.016), se confirma el equilibrio de los marcadores. Si bien existe una selección por manejo reproductivo para estos rebaños, sin embargo el equilibrio observado indicaría la ocurrencia de apareamientos entre individuos poco relacionados, con una presión selectiva mínima sobre la población, lo que sugiere que la estrategia de manejo utilizada en los rebaños tiene similar efecto sobre las frecuencias genotípicas a las esperadas bajo panmixia, sin evidenciar aún el efecto del apareamiento dirigido para los individuos del rebaño de Munay Paqocha.

Los valores de $\mathrm{F}_{\text {IS }}$ estuvieron cercanos a cero para todos los loci $(0,016)$, concordante a lo descrito por Agapito et al. (2008) para poblaciones de alpacas de Puno, Huancavelica y Junín $\left(\mathrm{F}_{\mathrm{IS}}=0,0141\right)$ y por La Manna et al. (2011) para la población de la Estación Experimental Quimsachata-Puno $\left(\mathrm{F}_{\mathrm{IS}}=\right.$ $0,01)$, pero con valores menores a los encontrados por Paredes et al. (2013) en poblaciones de Arequipa y Puno $\left(\mathrm{F}_{\mathrm{IS}}=0,15252\right)$. De Igual forma, los valores de $\mathrm{F}_{\mathrm{ST}} 0,003$ ) cercanos a cero (que explica el efecto Wahlund asociado a una reducción de heterocigosidad en una población causada por una estructura subpoblacional) indican la existencia de la poca diferenciación entre los rebaños. Los valores $\mathrm{F}$ fueron menores a los descritos en otras poblaciones de camélidos domésticos de Argentina, Bolivia y Perú (Barreta et al., 2012; Bustamante et al., 2006; Paredes et al., 2012). Estos resultados estarían asociados al tipo de manejo que ocurre en estos rebaños, basado en el intercambio de machos entre criadores.

A pesar de la presión de la selección reproductiva que existe en las poblaciones de estos rebaños reproductores, los individuos presentaron alto grado de variabilidad genética.

El conocimiento de la diversidad genética en poblaciones domésticas permite evaluar el estado actual de las mismas. En los últimos años se ha informado sobre la pérdida de la variabilidad de razas locales (Barker 1999; FAO, 2010), por lo que se recomienda diseñar planes de mejoramiento más adecuados para evitar la disminución de la diversidad genética y por ende la capacidad de tolerar a cambios ambientales, procurando de este modo la mejora en la producción pecuaria. En ese aspecto, la diversidad genética expresada como heterocigosidad esperada (He) descritas para el total de la población, guarda relación con los resultados obtenidos por Barreta et al. (2012) en alpacas de Bolivia, pero mayor $(\mathrm{He}=0.849+0.043)$ 
a lo informado tanto en trabajos anteriores para la especie (Agapito et al., 2008, La Manna et al., 2011, Paredes et al., 2011), así como en otras especies domésticas (Ema et al., 2014).

Por otro lado, los niveles de heterocigosidad observados (Ho) fueron inversos entre los rebaños de alpacas analizados. Es así que el rebaño del Fundo Itita presentó una mayor Ho $(0,858+0,059)$ respecto a la $\mathrm{He}(0,848+0,039)$, mientras que el rebaño del Centro Munay Paqocha presentó un menor valor de la Ho $(0,815+0,070)$ respecto a la He $(0,848+0,049)$. Esto también se reflejó en los valores de $\mathrm{F}_{\mathrm{IS}}$; siendo positivo para Munay Paqocha $(0,039)$ e indicando un déficit de heterocigotos, y negativo para Itita $(-0,012)$ indicando un exceso de estos. Estos altos valores registrados podrían ser el producto de la introducción constante de machos pertenecientes a diferentes acervos genéticos, de tal forma que generan una diversidad artificial y sobreestimada.

Si bien en este estudio no se detectaron altos niveles de endogamia y la mayoría de marcadores mostraron exceso de heterocigotos, el rebaño del Centro Munay Paqocha presentó una tendencia al déficit de heterocigotos, propenso a incrementar el nivel de endogamia, dependiendo del tipo de sistema de cruzamientos que se maneje en el centro. En este contexto, el mantener un bajo grado de endogamia en las poblaciones resulta importante ya que los altos niveles de consanguinidad van a afectar al fenotipo, y estar relacionada con la aparición de malformaciones congénitas y problemas reproductivos (Huanca et al., 2007).

Uno de los principales requerimientos para la aplicación de las estrategias de mejoramiento genético exitosas, es el conocimiento de la paternidad de los individuos a fin de generar registros genealógicos confiables. La precisión de la selección dependerá en gran medida de la calidad y cantidad de los registros de rendimiento y genealógicos disponibles. Igualmente, el establecimiento de un servicio de pruebas de paternidad resulta de utilidad para los criadores de ganado en la medida en que les permite resolver problemas asociados a la falta o incorrecta asignación de identidad de los animales pertenecientes a un hato. Así, el uso de marcadores microsatélites para estudios de paternidad en animales otorga un mayor grado de confianza en el diagnóstico, en comparación con los análisis de los grupos sanguíneos antes utilizados (Forbes, 1951), ya que brinda un gran poder de discriminación, con alta probabilidad de diferenciar a dos individuos como los posibles padres. En este sentido, los 10 marcadores utilizados en este estudio presentaron una probabilidad de exclusión adecuada, con un valor superior al $99,9 \%$ cuando se conoce el genotipo de ambos padres. Similares valores se obtienen en estudios de exclusión para poblaciones humanas, llamas, guanacos y bovinos (Bustamante et al., 2002; Martínez et al., 2005; Ossa et al., 2010; Montoya et al., 2010). De acuerdo a los valores de PE-2, se determina que se puede alcanzar un valor PEC de 0,999 con solo considerar los marcadores YWLL08, YWLL44, LCA37, YWLL36, LCA8; siendo YWLL08 el marcador por excelencia para este tipo de pruebas $(0,885)$. Entre más alto sean los valores PE2, mayor será la probabilidad de exclusión combinada para el marcador en cuestión (Marshall et al., 1998), y está relacionada al número de alelos por locus y PIC. Todas estas propiedades nos permiten proponer al panel de marcadores utilizados en este estudio como útiles para resolver casos de paternidad.

La prueba de filiación realizada se detecto mayores errores de asignación de maternidad y paternidad en el rebaño Fundo Itita, en comparación con el Centro de Munay Paqocha. Además, los porcentajes fueron mayores a los encontrados por Agapito et al. (2008) indicando un 15,5\% de error de paternidad y $4,44 \%$ error de maternidad. Estos errores de designación reflejan los problemas existentes en los rebaños ocasionados por la pérdida de aretes, el asignamiento basado en la cercanía de la cría a la supuesta madre, diferencias del sistema de apareamientos utilizadas entre los rebaños; es decir se refleja la falta de una infraestructura adecuada en el Fundo Itita que impide el control de los apareamientos de forma eficiente. Todos estos factores, al generar registros erróneos, pueden tener un gran impacto sobre la eficiencia de las pruebas de progenie y de la exactitud en la estimación de los valores de cría.

\section{CONCLUSIONES}

Los rebaños Munay Paqocha y el Fundo Itita mostraron altos $\mathrm{y}$ similares niveles de heterocigosidad, aunque con una tendencia al déficit de heterocigotos en el Centro Munay Paqocha y al exceso en Itita. La presencia de alelos únicos en los loci LCA37, LCA90, LCA5, VOLP92, YWLL36, YWLL44, YWLL08, permiten seleccionarlos 
con potencial aplicación para los estudios de diferenciación entre poblaciones de alpacas. Sin embargo, resulta importante la identificación de estos alelos también en otras especies de camélidos para poder seleccionar aquellos que determinen posibles introgresiones, y que permitan el monitoreo de una posible disminución de la variabilidad genética. Por otro lado, se puede obtener un acierto de paternidad al 99,9, considerando sólo 5 loci, con las mayores probabilidades de exclusión. El alto poder de discriminación y contenido polimórfico del marcador YWLL08 resulta útil para estudios de diversidad genética, identificación de individuos y determinación de paternidad en alpacas. Todos los marcadores presentaron altos valores de PIC sin embargo este valor es dependiente del numero de alelos y sus frecuencias, por lo que este parámetro no se considero como único aspecto para elegir o rechazar un marcador. El Fundo Itita presentó el mayor número de asignaciones maternas y paternas incorrectas respecto al centro Munay Paqocha, lo que demuestra la importancia de detectar estos errores en rebaños bajo selección para evitar la sobreestimación de valores genéticos cuantitativos. Finalmente, se resalta la importancia del monitoreo de la diversidad genética para el conocimiento de los cambios genéticos poblacionales y el efecto de la selección artificial en los rebaños ocurridos en el tiempo, así como el uso de las herramientas moleculares como apoyo a los programas de mejoramiento de las alpacas de Perú.

\section{AGRADECIMIENTOS}

Los autores agradecen al Programa FINCyT del Ministerio de la Producción del Gobierno del Perú. También a Matilde Riquelme, por las facilidades en la colecta de muestras en el SPAR Macusani.

\section{Correspondencia:}

Claudia Yalta

Clayama29@gmail.com

\section{REFERENCIAS BIBLIOGRÁFICAS}

1. Agapito J, Rodríguez J, Herrera-Velit P, Timoteo O, Rojas P, Boettcher P, García F, Espinoza J. 2008. Parentage testing in alpacas (Vicugna pacos) using semi-automated fluorescent multiplex PCRs with 10 microsatellite markers. Animal genetics. 39(2): 201203.

2. Barreta J, Iñiguez $\mathrm{V}$, Saavedra $\mathrm{V}$, Romero $\mathrm{F}$, Callisaya, A. M., Echalar J, Gutiérrez-Gil B., Arranz J. 2012. Genetic diversity and population structure of Bolivian alpacas. Small Ruminant Research, 105(1): 97-104.

3. Barker J. 1999. Conservation of livestock breed diversity. Animal Genetic Resources Information. 25: 33-43.

4. Briones I, Valdivia V. 1985. Defectos anatómicos en el camélido sudamericano doméstico. IDESIA (Chile). 9: 41-44.

5. Bustamante A, Zambelli A, De Lamo D, Von Thungen J, Vidal-Rioja L. 2002. Genetic variability of guanaco and llama populations in Argentina. Small Runimant Research. 44: 97-101.

6. Bustamante A, Maté L, Lamas E, Giovambattista G, Zambelli A, Vidal-Rioja L. 2006. Análisis de diversidad genética en tres poblaciones de llamas (Lama glama) del noroeste argentino. Revista chilena de historia natural. 79(2): 175-184.

7. Burgos W, Cerón M, Moreno M. 2010. Comparación de métodos para la extracción de ADN en cuyes (Cavia porcellus Rodentia, caviidae).Livestock Research for Rural Development. 22(81).

8. Chikhi L, Goossens B, Treanor A, Bruford M. 2004. Population genetic structure of and inbreeding in an insular cattle breed, the Jersey, and its implications for genetic resource management. Heredity. 92(5): 396-401.

9. Di Rocco F, Posik D, Ripoli M, Díaz S, Maté M, Giovambattista G, Vidal-Rioja L. 2011. South American camelid illegal traffic detection by means of molecular markers. Legal Medicine. 13(6): 289292.

10. Egito A, Paiva S, Maria Do Socorro M, Mariante A, Almeida L, Castro S, Grattapaglia D. 2007. Microsatellite based genetic diversity and relationships among ten Creole and commercial cattle breeds raised in Brazil. BMC Genetics. 8(1): 83.

11. Ema P, Manjeli Y, Meutchieyié F, Keambou C, Wanjala B, Desta A, Ommeh S, Skilton R Djikeng A. 2014. Genetic diversity of four Cameroonian indigenous cattle using microsatellite markers. Journal of Livestock Science. 5: 9-17.

12. FAO. 2005 .Situación Actual de los Camélidos Sudamericanos en Perú.

13. FAO. 2010. La situación de los recursos zoogenéticos mundiales para la alimentación y la agricultura, editado por Barbara Rischkowsky y Dafydd Pilling. Roma (disponible en http://www. fao.org/docrep/011/ a1250s/a1250s00.htm) (traducción de la versión original en inglés, 2007).

14. Forbes G. 1951. Blood Groups and Disputed Paternity. British Medical Journal. 2(4725): 227.

15. Frankham R, Briscoe D, Ballou J. 2002. Introduction to Conservation Genetics. United Kingdom: Cambridge University Press. 617p. ISBN 0-52163985-9 (paperback), 0-521-63014-2 (hardback).

16. Goudet J. 1995. FSTAT, version 2.9.3. A program to estimate and test gene diversities and fixation indices. Journal Heredity.(86) 487-488

17. Garber R, Morris J. 1983.General equations for the 
average power of exclusion for genetic systems of $n$ codominant alleles in one-parent and no-parent cases of disputed parentage. Inclusion Probabilities in Parentage Testing, 277-280.

18. Guo S, Thompson E. 1992. Performing the exact test of Hardy-Weinberg proportion for multiple alleles. Biometrics, 361-372.

19. Huanca T, Apaza N, Sapana R. 2007. Defectos congénitos y hereditarios visibles en alpacas de dos zonas representativas de la región de Puno. Archivos Latinoamericanos de Producción Animal. (15): 480.

20. Jianlin H, Taylor C. 2000. Application of New World Camelidae microsateIlite primers for amplification of polymorphic loci in Old World Camelids.Animal Genetics. 31: 404-419.

21. Kadwell M, Fernandez M, Stanley H, Baldi R, Wheeler J, Rosadio R, Bruford M. 2001.Genetic analysis reveals the wild ancestors of the llama and the alpaca. Proceedings of the Royal Society of London. Series B: Biological Sciences. 268(1485): 2575-2584.

22. Kalinowski S, Taper M, Marshall T. C. 2007. Revising how the computer program CERVUS accommodates genotyping error increases success in paternity assignment. Molecular ecology. 16(5): 1099-1106.

23. Lang K, Wang Y, Plante Y. 1996.Fifteen polymorphic dinucleotide microsatellites in llamas and alpacas. Animal Genetics. 27(4): 293-293.

24. La Manna V, La Terza A, Ghezzi S, Saravanaperumal S, Apaza N, Huanca T, Bozzi R, Renieri C. 2011. Analysis of genetic distance between Peruvian Alpaca (Vicugna pacos) showing two distinct fleece phenotypes, Suri and Huacaya, by means of microsatellite markers. Italian Journal of Animal Science. 10(60): 271-276

25. Laval G, Iannuccelli N, Legault C, Milan D, Groenen M, Giuffra E, Andersson L, Nissen P, Jorgensen C, Beeckmann P, Geldermann H, Foulley JL, Chevalet C, Ollivier L. 2000. Genetic diversity of eleven European pig breeds. Genetics Selection Evolution. 32(2): 187-204.

26. Marin J, Saucedo C, Corti P, González B. 2009. Application of DNA forensic techniques for identifying poached guanacos (Lama guanicoe) in Chilean Patagonia. Journal of Forensic Sciences. 54(5): 1073-1076.

27. Marshall T, Slate J, Kruuk L, Pemberton J. 1998. Statistical confidence for likelihood-based paternity inference in natural populations. Molecular Ecology. 7(5): 639-655.

28. Mburu D, Ochieng J, Kuria S, Jianlin H, Kaufmann B, Rege J, Hanotte O. 2003. Genetic diversity and relationships of indigenous Kenyan camel (Camelus dromedarius) populations: implications for their classification. Animal Genetics. 34(1): 26-32.

29. Martínez A, Vega-Pla J, Bravo M, Barba C, Caraballo J, Delgado J. 2005. Caracterización genética de la oveja Palmera con microsatélites. Archivos de
Zootecnia. 54(206): 363-367.

30. Montoya A, Cerón Muñoz M, Moreno M, Martínez E, Corrales J, Tirado J, Calvo S. 2010. Genetic characterization of the Hartón del Valle, Angus, Brangus, Holstein, and Senepol cattle breeds in Colombia, using ten microsatellite markers. Revista Colombiana de Ciencias Pecuarias. 23(3): 283-291.

31. Obreque V, Coogle L, Henney P, Bailey E, Mancilla R, Garcia-Huidobro J, Hinrichsenl P, Cothran E.G. 1998. Characterization of 10 polymorphic alpaca dinucleotide microsatellites. Animal Genetics. 29(6): 461-462.

32. Obreque V, Mancilla R, Garcia-Huidobro J, Cothran E, Hinrichsen P. 1999. Thirteen new dinucleotide microsatellites in Alpaca. Animal Genetics. 30(5): 397-398.

33. Ossa H, Tascón E, Moreno H, Horta J, Moreno G. 2010. Frecuencias alélicas de 14 STR's autosómicos en una población de Antioquia, Colombia. NOVA Publicación Científica. 8(13): 25-29

34. Paredes M, Membrillo A, Azor P, Machaca J, Torres D, Serrano A. 2013. Genetic and phenotypic variation in five populations of Huacaya Alpacas (Vicugna pacos) from Peru. Small Ruminant Research. 111(1-3): 31-40.

35. Pariset L, Savarese M, Cappuccio I, Valentini A. 2003. Use of microsatellites for genetic variation and inbreeding analysis in Sarda sheep flocks of central Italy. Journal of Animal Breeding and Genetics. 120(6): 425-432.

36. Penedo M, CaetanoA, Cordova K. 1998. Microsatellite markers for South American camelids. Animal Genetics. 29: 411-412

37. Penedo M, Caetano A, Cordova K. 1999. Six microsatellite markers for South American camelids. Animal genetics. 30(5): 399-399.

38. Rousset F, Raymond M. 1995. Testing Heterozygote Excess and Deficiency. Genetics. 140: 1413-1419.

39. Sambrook J, Russell D. W. 1989.Molecular Cloning: A Laboratory Manual. Vol. 3. Cold Spring Harbor Laboratory Press.

40. Schulz U, Minguez Y, Checa M, Garcia-Atance P, Dunner S, Garcia D, Cañón J. 2005. The Majorero camel (Camelus dromedarius) breed. Animal Genetic Resources. 36: 61-72.

41. Tolone M, Mastrangelo S, Rosa A, Portolano B. 2012. Genetic diversity and population structure of Sicilian sheep breeds using microsatellite markers. Small Ruminant Research. 102(1): 18-25.

42. Velarde J. Consanguinidad y su importancia en el mejoramiento genético de la alpaca. Sistema de Revisiones en Investigación. 2011. Veterinaria de San Marcos. Disponible en internet: http://www.unmsm. edu.pe/veterinaria/files/Articulo_vilela_velarde.pdf

Recibido 26/01/2015

Aceptado 03/02/2015 